\title{
Systematic reliability studies of back-contact photovoltaic modules
}

This paper has been presented at the SPIE Optics and Photonics Conference, 12-16 August 2012, San Diego, USA

\author{
V. Rosca \\ I.J. Bennett \\ W.E. Eerenstein
}

October 2012

ECN-M--12-041 


\title{
Systematic reliability studies of back-contact photovoltaic modules
}

\author{
Victor Rosca*, Ian J. Bennett, and Wilma Eerenstein, \\ ECN Solar Energy, PO Box 1, 1755 ZG, Petten, The Netherlands
}

\begin{abstract}
Back-contact module technology offers the advantage of lower yield loss, higher power conversion efficiency, and significantly faster manufacturing as compared to conventional H-pattern modules. In this paper we present results of a systematic accelerated ageing study of ECN back-contact metallization wrap through (MWT) modules. A series of fullsize $(6 \times 10$ cells) MWT modules based on combinations of four different conductive back-sheet foils, two encapsulants, and two electrically conductive adhesives were manufactured and subjected to the damp heat conditions as defined in the IEC61215 edition 2 standard. Modules that combine conductive back-sheet foil with certain types of isolation lacquer (also referred to as inner layer dielectric, ILD) and EVA showed a high failure rate. It appears that a combined effect of moisture and EVA causes a weakening of adhesion strength at $\mathrm{Cu} / \mathrm{ILD}$ interface and decisively contributes to delamination at $\mathrm{Cu} / \mathrm{ILD}$ interface. This delamination puts stress on the interconnection and ultimately results in interconnection failure. Removal of ILD significantly improves the stability of MWT modules in damp heat, as up to $2000 \mathrm{hrs}$ of testing only up to $2.4 \%$ relative power loss was observed, and also lowers the foil cost.
\end{abstract}

Keywords: back-contact PV modules, metallization wrap through technology, damp heat test, conductive back-sheet foil

\section{INTRODUCTION}

Power output of a PV module is determined by the cell efficiency as well as the optical and resistance losses in the module. The yield of the module manufacturing process is determined by the degree of cell breakage occurring, particularly during the interconnection process. In order to reach significant cost reduction for solar modules, the efficiency of the module must be increased, the material cost reduced and the process yield increased.

ECN Solar has developed a metallization wrap through (MWT) cell and back-contact module technology [1-4]. The back-contact module technology allows a single-step encapsulation and interconnection process of the back-contact cells. The main distinctive feature of this technology is use of a patterned conductive back-sheet foil and a conductive adhesive to make the electrical connection between the cells. This back-contact module technology offers the advantage of lower yield loss, higher power conversion efficiency, and significantly faster manufacturing as compared to conventional $\mathrm{H}$ pattern modules. ECN has obtained IEC61215 and IEC61730 certificates for this technology.

The main advantages of MWT cells and back-contact modules include reduced shadowing due to the absence of bus-bars and tabs at the front of the cells. The cells can be placed closer together in the modules as no tabs pass between the front and rear of the cell. The current carrying component of the module can be wider than conventional tabbing as there are no shadowing losses. Therefore higher cell efficiencies and module output can be achieved in MWT modules as compared to H-pattern modules.

Figure 1 shows a schematic drawing of the build-up of an MWT module and cross section of such a module after lamination. Conductive adhesive paste is stencil printed on the conductive back-sheet. The rear-side encapsulant sheet is then punched and placed on the back-sheet with the openings in the encapsulant corresponding to the position of the conductive adhesive. Cells are placed on the stack by a pick-and-place robot. This is the only cell-handling step; therefore, thinner and larger cells can easily be used with very low cell breakage rate. Finally, the front side encapsulant sheet and glass are placed. The complete stack is then inverted and laminated. The interconnection and encapsulation step are combined in a single lamination step as the conductive adhesive and encapsulant are chosen to have the same curing conditions. This module manufacturing process has been developed together with the Dutch equipment manufacturer Eurotron [1], and fully automated production lines for manufacturing of back-contact modules are now commercially available.

*rosca@ecn.nl; phone +31 88515 4612; fax +31 88515 8214; www.ecn.nl

Reliability of Photovoltaic Cells, Modules, Components, and Systems V, edited

by Neelkanth G. Dhere, John H. Wohlgemuth, Proc. of SPIE Vol. 8472, 84720D · (c)

2012 SPIE · CCC code: 0277-786/12/\$18 · doi: 10.1117/12.930441

Proc. of SPIE Vol. 8472 84720D-1 

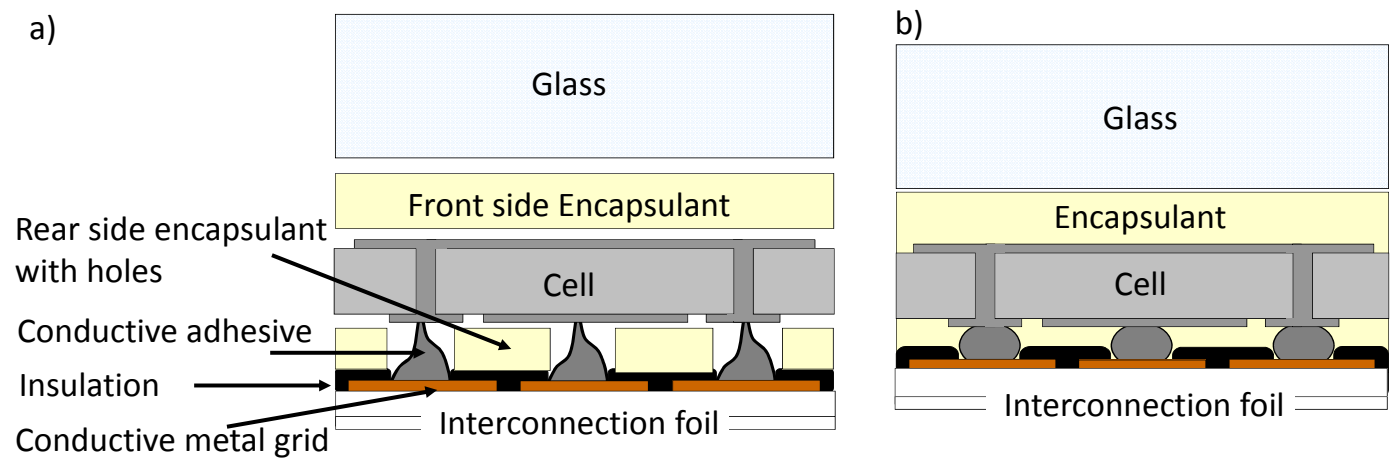

Figure 1. Schematic drawing of a) the build-up of an MWT back contact module with conductive back sheet foil and adhesive as interconnection and $b$ ) a cross section of such a module after lamination.

The conductive back-sheet foil consists of a polymer laminate (PVF-PET or alternative) with a Cu layer attached to it. The $\mathrm{Cu}$ layer is patterned to provide a series electrical connection between the cells. Patterning is currently done by wetchemical etching. An isolation layer (an UV- or temperature-cured lacquer referred to as inner layer dielectric, ILD) is applied to the patterned conductive back-sheet to prevent unwanted electrical contact between cell and the foil. The introduction of a new module technology based on a back-sheet foil with an integrated conductive grid in combination with conductive adhesive raises issues in terms of the reliability of the concept. Recent studies carried out on small-size (single and four cells) and full-size $(6 \times 10$ cells) modules pointed at a prominent impact of dimensional and functional stability of the conductive back-sheet foil on the module reliability [5,6]. Guichoux et al. [5] pointed at an interaction between encapsulant and ILD in the presence of moisture causing delamination and ultimately module failure. Eerenstein et al. [6] have reported on MWT modules successfully passing TC300, DH2000, and the wet leakage test. However, a few modules failed after $1000 \mathrm{hrs}$ in DH. A failure analysis based on dark lock-in thermography (DLIT) data and opening the modules pointed at an interconnection failure possibly related to delamination.

The damp heat test is perhaps the most critical test for MWT modules. Our recent studies on small-size modules based on foils with ILD pointed at a significant performance loss after $1000 \mathrm{hrs}$ in DH. Interestingly enough, better DH results were observed for small-size modules manufactured with foils without ILD. Figure 2 compares the effect of accelerated ageing in DH on the fill factor losses for 3 series of small-size modules and is meant to underpin the above observations. For modules based on foils without ILD an effect of the cell can also be inferred. However, these modules clearly perform much better in damp heat as compared to the modules based on foils with an ILD, irrespective of cell used.

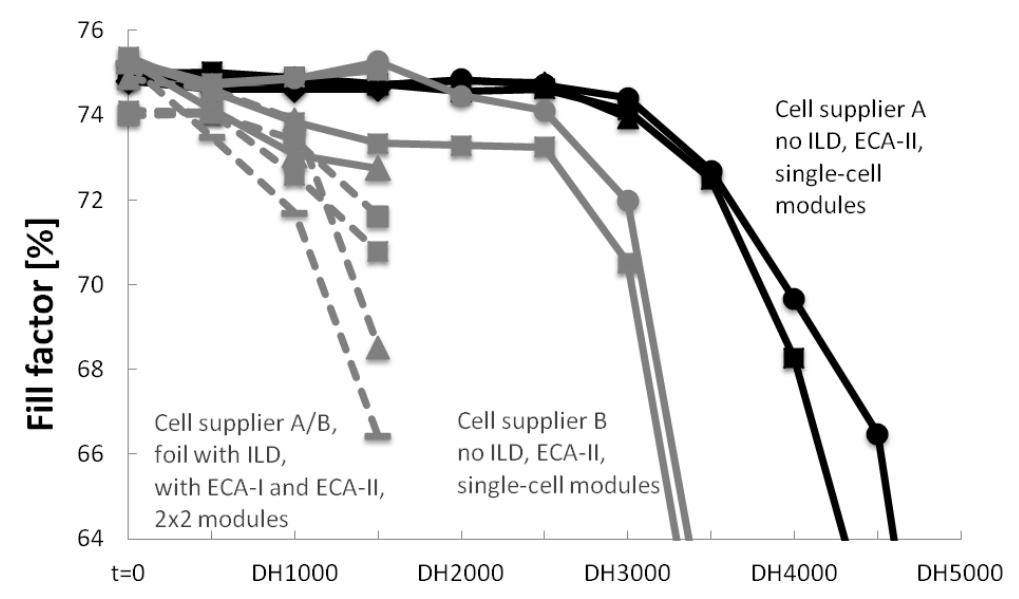

Figure 2. Effect of accelerated ageing in damp heat of a series of $2 \times 2$ modules based of foil with ILD01 and a series of single-cell modules based on foils without an ILD on the module fill factor. Cells of two different suppliers (A and B) were used in order to assess the effect of cell. 
In this paper we present results of an accelerated ageing study of ECN back-contact MWT modules. A series of full-size ( $6 \times 10$ cells) MWT modules based on combinations of four different conductive back-sheet foils, two encapsulants, and two electrically conductive adhesives (ECA) were manufactured and subjected to damp heat (DH) conditions $\left(85^{\circ} \mathrm{C}\right.$ and $85 \%$ relative humidity) as defined in the IEC61215 edition 2 standard. We present and discuss the results of this study with an emphasis on the impact of the conductive back sheet foil on reliability of the modules.

\section{EXPERIMENTAL}

Full-size modules were manufactured on a pilot back-contact module assembly line at ECN. For details on the module manufacture process see refs $[1,2]$. To test the compatibility between various module components under damp-heat conditions, modules with four different conductive back-sheets, two encapsulants, and two ECAs were built. See Table 1 for the list of modules and their composition. The MWT cells were part of a large batch purchased from a cell manufacturer. Cells were characterized by I-V testing and sorted according to their efficiency range.

Table 1 lists the modules manufactured and main components used in each $6 \times 10$ module. Four different back-sheet foils were chosen: two foils with different ILD type (ILD01 and ILD02); one foil with no ILD; and one foil with ILD (ILD01) and an Al layer integrated into the conductive back-sheet serving as a moisture barrier. These four foils were combined with two encapsulants; a standard cure EVA and a thermoplastic encapsulant (TP). Use of different ECAs was dictated by the type of the contact surface on the conductive back-sheet. All foils with ILD had silver-plated contact points and were used in combination with ECA-I. The ILD-free foil had no silver-plated contact points. Therefore, a different ECA (ECA-II) was used to ensure good electrical connection with bare $\mathrm{Cu}$.

The modules were characterized at ECN (I-V measurements, electroluminescence (EL), dark lock-in thermography (DLIT)) before and after exposure to DH for $2000 \mathrm{hrs}$. Climatic chamber testing and characterization (I-V, EL) was performed at Photovoltaik Insitut Berlin (PI Berlin, Germany), where measurements were also performed after $1000 \mathrm{hrs}$.

Table 1. Experimental matrix and list of modules manufactured and tested in this work.

\begin{tabular}{lccc}
\hline Code module & Conductive back sheet foil & Encapsulant & Electrically conductive adhesive \\
\hline $\mathrm{A} 800, \mathrm{~A} 801$ & PVF-PET-Cu-ILD01 & EVA & ECA-I $^{3}$ \\
\hline $\mathrm{A} 802, \mathrm{~A} 803$ & PVF-PET-Cu-ILD02 & EVA & ECA-I \\
\hline $\mathrm{A} 804, \mathrm{~A} 805$ & PVF-PET(Al)-Cu-ILD01 & EVA & ECA-I \\
\hline $\mathrm{A} 806, \mathrm{~A} 807$ & PVF-PET-Cu-ILD01 & TP & ECA-I \\
\hline $\mathrm{A} 808, \mathrm{~A} 809$ & PVF-PET-Cu-ILD02 & TP & ECA-I \\
\hline $\mathrm{A} 810, \mathrm{~A} 811$ & PVF-PET(Al)-Cu-ILD01 & TP & ECA-I \\
\hline $\mathrm{A} 812, \mathrm{~A} 813$ & PVF-PET-Cu (no ILD) & EVA & ECA-II $^{2}$ \\
\hline $\mathrm{A} 814, \mathrm{~A} 815$ & PVF-PET-Cu (no ILD) & TP & \\
${ }^{1}$ This foil contains an Al layer in the PVF-PET back sheet and serves as a moisture barrier. \\
${ }^{2}$ TP - thermoplast \\
${ }^{3}$ ECA-I was used in combination with foils with ILD. These foils had Ag-plated contacts applied to copper. \\
${ }^{4}$ ECA-II was used in combination with foils without ILD and was applied directly on Cu. \\
\hline
\end{tabular}

\section{RESULTS AND DISCUSSIONS}

Table 2 shows the I-V data acquired under standard test conditions after the module manufacture. The module output and the calculated encapsulated cell efficiency were in good agreement with the efficiency range of cells used. Accordingly, the cell-to-module losses were comparable for all modules $(0.3 \pm 0.1 \%$ on average). This is an indication that the choice of components and/or their combinations did not significantly affect the cell-to-module losses at zero hours.

Figure 3 summarizes the results of the accelerated ageing of MWT modules in damp heat. Three modules failed in the first 1000 hours in DH (A800, A801, A802) and one failed after 1000 hours in DH (A803). These four modules contained foils with ILD (either ILD01 or ILD02) and EVA as encapsulant (see Table 1). For the modules based on other materials combinations, all but one (A812) showed a relative loss in power output under $2 \%$ after 2000 hours in DH. Module A812 showed a relative loss of ca. $2.4 \%$ after 2000 hours DH. 
Table 2. Results of the I-V measurements under standard test conditions at $\mathrm{t}=0$.

\begin{tabular}{ccccccc}
\hline $\begin{array}{c}\text { Module } \\
\text { code }\end{array}$ & Pm[W] & Isc[A] & Voc[V] & FF[\%] & $\begin{array}{c}\text { Encapsulated } \\
\text { cell eff. [\%] }\end{array}$ & $\begin{array}{c}\text { Avrg. cell eff. } \\
\text { before } \\
\text { encapsulation[\%] }\end{array}$ \\
\hline $\mathrm{A} 800$ & 243.95 & 8.631 & 37.503 & 75.36 & 16.71 & 16.9 \\
\hline $\mathrm{A} 801$ & 244.83 & 8.642 & 37.562 & 75.42 & 16.77 & 17.0 \\
\hline $\mathrm{A} 802$ & 245.69 & 8.633 & 37.576 & 75.74 & 16.83 & 17.1 \\
\hline $\mathrm{A} 803$ & 245.63 & 8.633 & 37.544 & 75.78 & 16.82 & 17.1 \\
\hline $\mathrm{A} 804$ & 243.85 & 8.643 & 37.615 & 75 & 16.7 & 17.2 \\
\hline $\mathrm{A} 805$ & 247.15 & 8.655 & 37.671 & 75.8 & 16.93 & 17.2 \\
\hline $\mathrm{A} 806$ & 246.35 & 8.652 & 37.354 & 76.23 & 16.87 & 17.3 \\
\hline $\mathrm{A} 808$ & 246.88 & 8.678 & 37.436 & 76 & 16.91 & 17.3 \\
\hline $\mathrm{A} 810$ & 247.12 & 8.686 & 37.493 & 75.88 & 16.92 & 17.3 \\
\hline $\mathrm{A} 811$ & 247.36 & 8.682 & 37.488 & 76 & 16.94 & 17.4 \\
\hline $\mathrm{A} 812$ & 246.86 & 8.668 & 37.635 & 75.67 & 16.91 & 17.4 \\
\hline $\mathrm{A} 813$ & 248.5 & 8.681 & 37.679 & 75.97 & 17.02 & 17.6 \\
\hline $\mathrm{A} 814$ & 249.9 & 8.693 & 37.595 & 76.47 & 17.11 & 17.17 \\
\hline $\mathrm{A} 815$ & 250.76 & 8.715 & 37.67 & 76.38 & & \\
\hline
\end{tabular}

Failed modules A800, A802, and A803 were subjected to post-mortem inspection. No visual changes on the module front side could be found. Inspection of the back side of the failed modules revealed some signs of delamination of the conductive back-sheet. Delamination had a local character and covered 3 to 5\% of the back side at most. Opening of the modules at the suspected areas pointed at adhesive fracture at ILD/Cu interface as the main failure mode of the foil.

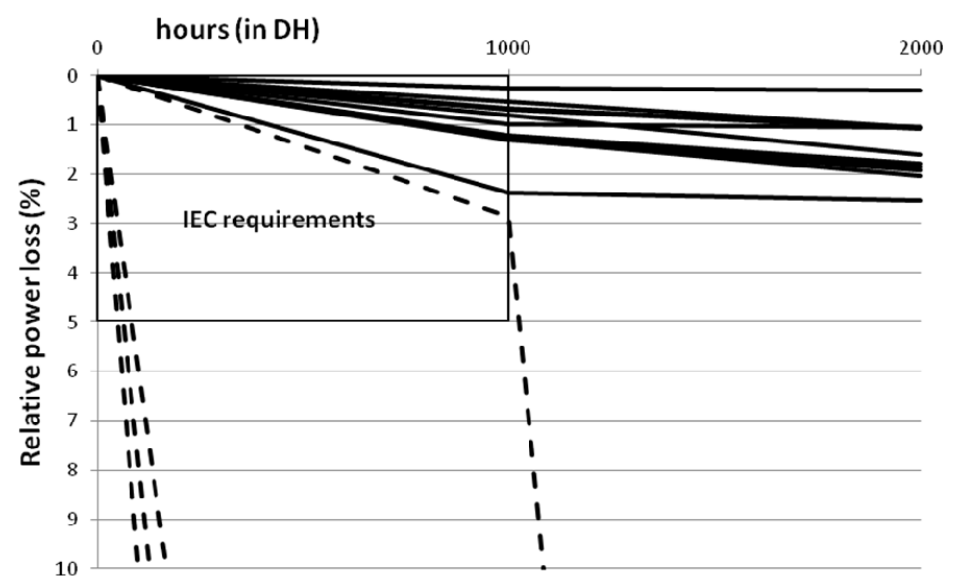

Figure 3. Effect of the accelerated ageing in damp heat on the relative power loss for a series of $6 \times 10$ MWT modules (see Table 1 for module composition). Data for four modules that failed before or after $1000 \mathrm{hrs}$ in DH is given by dash lines. These were modules A800, A801, A802, and A803.

The above results point at delamination at the ILD/Cu interface as the most likely cause of the module failure. As such a delamination was observed neither for modules that contained an alternative encapsulant (thermoplast) nor for modules that had a moisture barrier in the back sheet (Al layer), this delamination occurs due to a combined effect of EVA and moisture. The exact rate and extent of delamination probably depends on the moisture ingress and accumulation rate, EVA acidity, and perhaps initial stress/strain in the isolation lacquer and or polymeric backing (can depend on processing/batch differences). In any case, delamination at $\mathrm{Cu} / \mathrm{ILD}$ interface put mechanical stress on the interconnection, which might cause loss of mechanical and electrical contact between the foil and the cell contacts.

The adhesion between $\mathrm{Cu}$ and ILD could be time dependent. As learned from systematic peel tests carried out on small laminates that simulate the module (data not shown), adhesion between ILD (01 or 02) can be very low if measured right after DH test, but the adhesion improves as the sample dried out. Moreover, the fracture mode could change from adhesive right after DH test to cohesive (in ILD) after a few weeks time. This effect should be taken into account when 
investigating and improving the foil reliability. Furthermore, ECAs generally show good adhesion to both foil and cells. Different ECAs could show different adhesion strength and/or mechanical properties (elasticity), and therefore different resistance to the stress/strain.

Delamination at $\mathrm{Cu} / \mathrm{ILD}$ interface and module failure can be prevented in a number of ways. These include prevention of moisture ingress in to the module by use of moisture-blocking $\mathrm{Al}$ back sheet. The second method is to use an alternative to EVA as the encapsulant. Thermoplastic encapsulants in combination with moisture do not show delamination of the back-sheet. The final option is to remove the ILD from the back sheet. Indeed, the rear side encapsulant sheet seems to be able to provide sufficient electrical isolation between cell and the back sheet. Omission of ILD would obviously eliminate the issues related to delamination at $\mathrm{Cu} / \mathrm{ILD}$ interface. Note that foils with ILD normally contain silver-plated contact dots. These are meant to minimize the contact resistance between foil and (Ag-based) ECA. There are ECAs suitable for contacting directly on $\mathrm{Cu}$; so, application of additional contact dots is not required. Elimination of both ILD and the silver contact dots would simplify the foil manufacturing process and would allow a significant materials cost reduction.

\section{CONCLUSIONS}

In this paper we presented results of an accelerated ageing study of ECN back-contact MWT modules. A series of fullsize $(6 \times 10)$ MWT modules based on combinations of four different conductive back-sheet foils, two encapsulants, and two electrically conductive adhesives were manufactured and subjected to the damp heat conditions as defined in the IEC61215 edition 2 standard. Modules that combine conductive back-sheet foil with ILD and EVA showed a high failure rate. It appears that the combined effect of moisture and EVA causes a weakening of adhesion strength at $\mathrm{Cu} / \mathrm{ILD}$ interface. The resulted delamination at $\mathrm{Cu} / \mathrm{ILD}$ interface puts stress on interconnection and ultimately results in interconnection and module failure. Replacing EVA with an alternative encapsulant (e.g., a thermoplast) and/or use of a moisture barrier in the back sheet improves the module stability in damp heat. However, removal of ILD is the most interesting approach, as this not only significantly improves the stability of MWT modules in damp heat, but also lowers the foil cost.

\section{REFERENCES}

[1] Spath, M., De Jong, P.C., Bennett, I.J., Visser, T.P., Bakker, J., "A novel Module assembly line using back contact solar cells," Proc. 33rd IEEE Photovoltaic Specialist Conference, San Diego, USA, (2008).

[2] Bennett, I.J., Tjengdrawira, C., Mewe, A.A., Lamers, M.W.P.W., De Jong, P.C., Weeber, A.W., "World record module efficiency for large and thin mc-Si MWT cells," Proc. 24th European Photovoltaic Solar Energy Conference and Exhibition, Hamburg, Germany, (2009).

[3] Tjengdrawira, C., Lamers, M.W.P.W., Bennett, I.J., P.C. de Jong, World first 17\% efficient multi-crystalline silicon module, 35th IEEE Photovoltaic Specialist Conference, (2010).

[4] Eerenstein, W., Bennett, I.J., Spath, M., Dekker, N.J.J., Rosca, V., "Back contact module technology", China PV Technology International Conference, (2012).

[5] M. Guichoux, C. Tjengdrawira, D. Veldman, De Jong, P.C., "Impact of materials on back-contact module reliability," 35th IEEE Photovoltaic Specialist Conference, Honolulu, Hawaii, USA, (2010).

[6] Eerenstein, W., Bennett, I.J., Veldman, D., Visser, T.P., Brockholz, B., De Jong, P.C., Copetti, C.A., Wijnen, P., "Climate chamber test results of MWT back contact modules", 25th European Photovoltaic Solar Energy Conference and Exhibition, Valencia, Spain, (2010). 
